\title{
Prediction of breast cancer metastasis by genomic profiling: where do we stand?
}

\author{
Ulrich Pfeffer · Francesco Romeo · Douglas M. Noonan • \\ Adriana Albini
}

Received: 18 February 2009/Accepted: 12 March 2009/Published online: 24 March 2009

(C) The Author(s) 2009. This article is published with open access at Springerlink.com

\begin{abstract}
Current concepts conceive "breast cancer" as a complex disease that comprises several very different types of neoplasms. Nonetheless, breast cancer treatment has considerably improved through early diagnosis, adjuvant chemotherapy, and endocrine treatments. The limited prognostic power of classical classifiers determines considerable over-treatment of women who either do not benefit from, or do not at all need, chemotherapy. Several gene expression based molecular classifiers (signatures) have been developed for a more reliable prognostication. Gene expression profiling identifies profound differences in breast cancers, most probably as a consequence of different cellular origin and different driving mutations and can therefore distinguish the intrinsic propensity to metastasize. Existing signatures have been shown to be useful for treatment decisions, although they have been developed using relatively small sample numbers. Major improvements are expected from the use of large datasets, subtype specific signatures and from the re-introduction of functional information. We show that molecular signatures encounter clear limitations given by the intrinsic probabilistic nature of breast cancer metastasis. Already today, signatures are, however, useful for clinical decisions in specific cases, in particular if the personal inclination of the
\end{abstract}

\footnotetext{
U. Pfeffer · F. Romeo

Functional Genomics, National Cancer Research Institute, Genoa, Italy

D. M. Noonan · A. Albini $(\bowtie)$ IRCCS Multimedica, Milan, Italy

e-mail: adriana.albini@multimedica.it

D. M. Noonan

Univerisity Of Insubria, Varese, Italy
}

patient towards different treatment strategies is taken into account.

Keywords Breast cancer - Gene expression profiling Metastasis · Stroma · Angiogenesis

\section{Introduction}

Gene expression of a tumor is a complex intermediate phenotype that is determined by many different factors: (1) the host genotype, (2) eventual somatic mutations and genomic alterations, (3) the cell from which the tumor originated, and (4) the specific microenvironment in terms of stroma cells, infiltrating cells and cell signaling molecules. It is reasonable to assume that any difference between two tumors in their patho-physiological state should be represented by some difference in gene expression. This is true despite the fact that while genes and the messenger RNAs derived from them are not the executers of biological function, they can nonetheless be used as markers of it. Even events that are completely independent of gene transcription such as protein modifications, as a part of the cellular program, elicit a transcriptional response that can be detected by expression profiling.

However, tumors are heterogeneous and the different cell populations contained in different areas of the same tumor might well differ for their potential to progress and metastasize. The multistep carcinogenesis model predicts that tumors evolve through the consecutive acquisition of genetic and epigenetic alterations towards a more and more aggressive phenotype inasmuch as they acquire the characteristics needed for progression [proliferation, resistance to apoptosis, scattering, migration and invasion, stimulation of vessel growth, survival in the blood stream (anti- 
anoikis), adhesion to the vessel wall, extravasation, survival and growth in the target tissue] through random mutation and epigenetic changes followed by selection of the cells that carry a growth advantage in the specific environment [1-5]. One of the predictions of the multistep carcinogenesis model is that the "metastatic phenotype" might be a characteristic of only a very small subpopulation of the cells within the primary tumor and that the metastasizing cells could acquire additional mutations that are not present in the primary tumor $[2,6]$. These features would escape from detection by expression profiling (or any other genomic technique such as comparative genome hybridization on arrays) unless one analyzes each potentially unique cell population within a tumor, an impossible enterprise. Another recent theory predicts that metastasis arises from cancer "stem cells", better defined as "cancer initiating cells" that might stay latent in the primary tumor and escape to metastasis when local conditions, for instance in hypoxia, induce their scattering. In retrospect, the success of metastasis prediction by expression profiling of the primary tumor was not so obvious and it is still not very clear how this can be reconciled with the multistep carcinogenesis model [7-10].

The current view of carcinogenesis considers the tumor cells themselves as main actors in carcinogenesis. Mutations of oncogenes and tumor suppressor genes initiate and promote the development of the tumor, hence these mutations determine its fate. From this point of view, the analysis of the actual tumor cells instead of the whole tumor that also contains many host fibroblasts, endothelial cells, and blood cells, would be expected to yield the best information on the molecular status of the tumor. The presence of host cells was expected to dilute the signal derived from the mutated tumor cell population [11].

However, the importance of the tumor stroma and of its interactions with the neoplastic cells are increasingly recognized as important, if not determining, factors in the evolution of a tumor [12-17]. This is also supported by evidence that gene expression profiling of the entire tumor yields reliable signatures. We have recently shown that many of the genes contained in the metastasis signature developed by comparing many tumors of different tissues and metastases of unmatched tumors [18] are expressed by stromal fibroblast or endothelial cells. Genes encoding proteins of the extracellular space, which are produced at least in part by stromal cells, can be used to construct a prognostic signature [19]. Stroma cells, long believed to be passive by-standers, can undergo mutations that drive them to contribute to tumor progression: p53 mutations, for instance, have been detected in stromal fibroblasts in $40 \%$ of the tumors analyzed, prevalent even in the absence of p53 mutations in the tumor epithelial cells, and these mutations predicted lymphnode metastasis [20].
For the purpose of prognosis, it appears therefore useful or even necessary to screen all the tumor compartments for potential markers and not only the tumor cell itself.

For breast cancer, prognostic signatures are expected to reduce the tendency to apply what has been seen as overtreatment. Following the existing guidelines, adjuvant chemotherapy is offered to most breast cancer patients. Many of these patients have tumors that have a low risk of developing metastases or that do not respond to existing chemotherapies. A more precise prognosis of the risk of relapse and a response prediction are needed.

\section{Technical issues}

Microarray technology has developed to considerable maturity. State of the art platforms and protocols guarantee high reproducibility and a low intrinsic variability. It has been shown that most of the variability in microarray experiments derives from the biological sample itself, even when conducted under apparently identical conditions. The second most important source of variability is introduced by the operator whereas the intrinsic oscillation of present microarrays is negligible [21]. Also, in our experience, the correlation between gene expression data generated by microarray and those obtained by more classical approaches such as real time PCR is excellent. Most differences can eventually be attributed to differences in the region of selection of probes and primers (U. Pfeffer, unpublished observation) since alternative splicing appears to be more the rule than the exception. The same applies for cross-platform variation of microarray data [22]. The latest addition to the technological battery are arrays that contain several probes for each single exon of each single gene of the human genome and are expected to yield additional information on alternative splicing and variant expression as well as more robust data [23]. So far, no large breast cancer studies using these arrays have published.

The sensitivity of microarrays has grown continuously and the amount of tissue needed to perform the analysis has been greatly reduced. Latest generation microarray analyses start with $100 \mathrm{ng}$ total RNA that can be isolated from very small amounts of tissue. Analysis of material obtained from fine needle biopsies is therefore possible, potentially allowing for pre-operative diagnostic approaches [24-26]. However, the smaller the sample, the higher the risk for sampling non-representative areas of a typically heterogeneous tumor. The ratio between tumor epithelial cells and stroma cells sampled also varies in fine needle aspirates. For this reason, whenever possible, larger pieces that have accurately been selected by the pathologist should be used. Sample conservation remains critical for the success of 
expression profiling applications. Yet the laborious and logistically demanding procedure of liquid nitrogen freezing of intra-operative samples can be substituted by conservation in RNA inhibiting pre-fixation solution such as RNAlater [27] or HOPE [28] that also facilitate longterm storage thereby reducing the costs and eventually increasing stability of biological molecules. Data on the involvement of microRNAs in breast cancer are growing and therefore sample conservation and RNA extraction should also consider the need to isolate small RNAs, since not all miRNA screening procedures accept total RNA as a starting material.

A major concern has been the virtually absent overlap of signatures produced using different platforms. Unfortunately, not a single study to date has hybridized a large number of samples to two different platforms. It is therefore difficult to conclude to which degree the different platforms actually reveal the same gene expression events. The lack of overlap can probably be attributed mainly to different patient populations analyzed, which greatly determines the genes selected for the signatures, as discussed below.

\section{Breast cancer subtypes}

Microarray gene expression profiling reliably yields the complex intermediate phenotype of gene expression of a sample. The gene expression phenotype of a tumor reflects its past history in terms of cell origin, its present in terms of exposure to oxygen and nutrients, growth factors and hormones, inflammation and tumor infiltrate which in turn are believed to determine its future potential to develop metastases. The first indication of the power of gene expression profiling with microarray came from the analysis of tumors sampled before and after chemotherapy and, in two cases, of matched lymphnode metastases [29]. This work showed the existence of molecularly defined subgroups. Gene expression patterns in two tumors from the same individual were almost always more similar to each other than to any other sample. Additional studies have confirmed that primary tumors and their metastases share similar expression profiles [30, 31] although several genes that are differently expressed have been identified $[32,33]$.

Sorlie et al. [34] showed that breast cancer cases fall into two large molecular classes that correspond almost perfectly to the status of estrogen receptor expression as analyzed by ligand binding assays or immunohistochemistry. A bioinformatic analysis of the two classes revealed that they correspond to profoundly different tumors. Even after the stepwise removal of several hundreds of the genes that best discriminate between the two classes, almost all cases were still correctly assigned to the estrogen receptor $\alpha$ positive or negative classes of tumors [35]. The difference between these two classes is consistent with the hypothesis that the two different tumor types derive from different progenitor cells. For this reason, the two classes are also defined as luminal and basal type. However, gene expression profiling reveals additional subtypes that are reproducibly formed after hierarchical clustering of breast cancer datasets containing more than hundred cases (hence containing a sufficient number of cases for each subtype class) $[34,36]$. The estrogen receptor $\alpha$ positive type is subdivided into luminal A, luminal B and eventually C clusters and the estrogen receptor $\alpha$ negative type contains the basal-like, her2-like, and a "normal like" cluster. A similar classification has independently been obtained by Sotiriou et al. [36]. A more recent elaboration of breast cancer microarray expression profiles has confirmed the subtypes identified before [37]. The main determinant of subtypes remain HER2 and estrogen receptor status [38, 39]. The analysis by Hu et al. [37] tentatively names one of the estrogen receptor $\alpha$ negative subtypes "IFN-like" since STAT1 and several interferon induced genes are among the master discriminators of this cluster. Analyses of this type point to the identification of genes or pathways whose differential expression characterizes single clusters. This is particularly evident for the HER2 subtype. Tumors that cluster in this subtype show over-expression of HER2/neu/ ERBB2, the EGF receptor family member whose amplifications determine responsiveness to the humanized antibody trastuzumab, which is directed against the HER2 encoded protein [40, 41]. Similarly, more defined subclusters have been identified as being formed by tumors harboring BRCA1 and BRCA2 mutations, and a closely related subcluster shows alterations downstream of the two hereditary breast cancer genes without having the genes themselves mutated [42, 43]. It is most likely that, step by step, information on the molecular nature of the tumors clustering together will be obtained for most of the subclusters. Intriguingly, not all tumors belonging to a subcluster must necessarily express the master discriminator gene(s) nor is expression of these genes sufficient for assigning a single case to a specific subcluster. In fact, HER2 expressing tumors are found among nearly all the subtypes [34]. This indicates that tumors of any subtype can over-express HER2 without loosing their identity, but in this case, HER2 over-expression most probably has only a minor role in the etiology of the specific tumor and hence a lower potential as a response marker.

Molecular subtypes have a clinical relevance inasmuch as they have different propensities to metastasize. The genes that characterize the different subtypes form the intrinsic subtype signature that can prognosticate clinical behavior [34, 36, 37, 44]. Its development as a real time PCR based test has been reported [45]. 
The major classes of estrogen receptor $\alpha$ positive and negative breast cancers most probably are derived from different tumor progenitor cells. Weinberg et al. [46] have recently shown that the cell of origin is a major determinant of the propensity to metastasize. It cannot be excluded, at present, that the molecular subtypes identified correspond to different progenitor cells downstream to ER $\alpha+$ (luminal) or $\mathrm{ER} \alpha-$ (basal) progenitor cells. More likely, subtypes correspond to tumors sharing the same driving mutation or at least the same affected pathway, as it is evident for the HER2 and BRCA1 and 2 cases. Further studies on molecular characteristics will eventually identify pathway mutations and lead to a more profound understanding of etiological events in breast cancer carcinogenesis.

\section{Signatures}

The identification of molecular subtypes with clinical relevance indicated that it should be possible to develop prognostic signatures with an elevated accuracy in classifying tumors into risk groups that deserve differential treatment or eventually no treatment at all. This endeavor has been undertaken by several groups. The general approach is a retrospective analysis of deep frozen breast cancer specimens with known follow-up, selection of discriminator genes by a variety of bioinformatics and statistical methods, and the construction of a predictive multigene classifier on the base of the follow-up information available. The statistical aspects of this approach have been the argument of a survey of these kinds of studies where many flaws in the experimental design were revealed [47]. Essentially, two datasets are needed: one to develop the classifier (training set) and another to test the classifier (test or validation set). These two sets can be obtained by a random split if the original dataset is large enough. In this case, a validation on a third, completely independent set is desirable. In alternative, signatures can be developed by the cross-validation method where single cases are left out from the training set in an iterative manner. The cases left out are then classified in order to validate the method.

A major problem with existing sample collections is that most of the women with breast cancer have received adjuvant chemotherapy. Therefore, a tumor that later developed a metastasis is of certain metastatic potential, whereas a tumor that did not metastazie could be a tumor without metastatic potential, a tumor that has not yet yielded a metastasis but will eventually do so, or a potentially metastatic tumor that has responded to adjuvant chemotherapy. A further complication is that breast cancers can give metastases even numerous years after removal of the primary tumor. This is best taken into account by working with cases with a very long follow-up. The metastasis-free cases that represent response to chemotherapy are most probably limited to a relatively small group of cases. However, these cases will participate in determining the signature. In the theoretical case of a cohort entirely composed of tumors with metastatic potential but with half of these being responsive to chemotherapy, these latter would be classified as "low risk". This aspect could be dealt with by using cases that have not received adjuvant chemotherapy. However, with current standards of care such cases are rare and are becoming progressively rarer.

A variety of signatures have been developed and have been recently exhaustively reviewed [48-55]. Two of these signatures have already been developed for commercialization as centralized laboratory tests and are presently being tested in large prospective trials. The Oncotype DX ${ }^{\circledR}$ recurrence score has been developed as real time PCR assay that can be performed on formalin fixed paraffin embedded (FFPE) tissues [56]. The selection of genes that form the classifier was based on three preliminary studies using real time PCR on FFPE samples. The most robust and informative genes were incorporated into a 16 gene classifier and analyzed in comparison to five housekeeping genes. The test is directed towards estrogen receptor $\alpha$ positive lymphnode negative cancers and yields a continuous prognostic score and predicts benefit from tamoxifen (in the low and intermediate risk group) and from adjuvant chemotherapy (in the high risk group). The test has recently been included in the recommendations of the American Society of Clinical Oncology. A prospective trial (Trial Assigning Individualized Options for TreatmentTAILORx) with a planned accrual of at least 10,000 patients intends to answer the question of whether intermediate risk patients benefit from chemotherapy [57]. The genes tested mainly monitor ER and HER2 status and proliferation.

Laura van't Veer et al. [58, 59] from the Netherlands Cancer Institute in Amsterdam developed a 70-gene classifier (commercialized as MammaPrint ${ }^{\circledR}$ ) based on twocolor microarray technology (initially using Rosetta Inpharmatics inkjet microarray technology and subsequently Agilent's microarray platform). The signature was built using the cross validation leave-one-out procedure on 78 informative lymph node negative cases and was subsequently validated on a partially overlapping cohort of 295 lymph node negative and positive cases. The classifier assigns cases to poor and good prognosis groups. The comparison of the 70 gene classifier with the NIH and St. Gallen criteria shows that the genomic approach clearly outperforms both. One study claimed, however, that this classifier works comparably to the Nottingham Prognostic Index (NPI) [60]. An additional validation study revealed a more than $90 \%$ chance of being free of disease after 
5 years for patients classified in the low risk group. This signature is being prospectively validated in the Microarray in Node-Negative Disease May Avoid Chemotherapy (MINDACT) trial where the enrollment of 6,000 patients is planned [61]. Patients discordantly scored by the classical Adjuvant! Online (www.adjuvantonline.com) and the genomic MammaPrint ${ }^{\circledR}$ assay will be randomized to receive or not receive chemotherapy. This trial will yield microarray data from a very large cohort that will also contain many cases that, according to the genomic classifier, did not receive chemotherapy. The use of these data will certainly allow major improvements of existing molecular signatures.

Other genomic prognostic classifiers such as the 76-gene classifier (Rotterdam signature) [62], the wound healing signature [63] and the invasive gene signature [64] have been developed. In a more straightforward approach the group of Sotiriou and colleagues has given the long sought answer to the question whether grade 2 cancers are an independent entity or whether they incorporate features of grade 1 and 3 cancers. A 97-gene (128 probe sets) discriminator was built on Affymetrix HU133A arrays using cancers of pathological grade 1 and 3. This discriminator was then applied to grade 2 cancers that showed either a grade 1 or 3 profile rather than an intermediate expression phenotype [65]. These signatures are also being developed towards commercialization [66].

The overlap of genes contained in these signatures is very low. However, the main determinants of all the signatures are proliferation, ER-status, HER2-status and, less prominently, angiogenesis, invasiveness and apoptosis. Most probably these signatures detect the same biological processes and pathways involved in metastasis. Depending on the actual patient cohort, the assay platform and the analytic algorithms used, different genes are selected for the signature. This is also supported by a bioinformatics analysis that shows the possibility to select other equally informative 70 gene classifier sets from the data set used by Van't Veer and Bernards [67]. This most probably indicates that many genes, probably several hundred or even more than one thousand, are actually related to disease free survival, and that the genes selected for any one classifier heavily depend on the patient cohort. In an additional study, the same authors calculated that for the development of a stable, cohort-independent classifier, several thousands of patients are needed [68].

A validation of several signatures (intrinsic subtype, 70gene classifier, wound healing signature, recurrence score and two gene classifier) revealed that with the exception of the latter, all signatures have a similar discriminating power showing 77 to $80 \%$ agreement in outcome scoring. Importantly, the combination of the signatures did not perform better, again indicating that the different signatures actually identify the same molecular characteristics by using different marker genes [44].

This, however, also means that a considerable part of cases is misclassified by any and all of the signatures. This raises the question of whether the existing signatures can be improved to yield a more reliable prediction of outcome. This is of particular importance if one keeps in mind that the classifier is to be used to withhold patients from chemotherapy. In this context, misclassification means not to treat a woman who might have benefited from chemotherapy. This is also important since this aspect determines the major skepticism among the oncologists who must decide whether or not to base the treatment decision they offer to the patients on molecular signatures.

\section{Limits of molecular signatures}

In order to explore the possibility of compiling the ultimate signature we have performed a simple simulation based on a publicly available breast cancer dataset (Gene Expression Omnibus, GEO1456) of 159 consecutive breast cancer cases from Stockholm with follow-up information of at least 8 years [69]. Forty of the 159 cases developed distant metastases. We have clustered these 159 cases using several gene lists that were obtained by selecting genes contained in specific Gene Ontology categories [70] that were associated with the parameter "relapse" with a $P$ value below 0.001 . The Gene Ontology terms "cell cell signaling", "cell death", "cell growth", "cell proliferation", "kinase", "metabolism", "signal transduction", "transcription regulator activity" were selected since they stand for different functional aspects that are involved in the process of metastasis. We then used the genes selected from each category separately for hierarchical clustering of the samples. This procedure necessarily produces highly discriminating gene lists since the classifier is developed on the same dataset to which it is applied. For the development of a molecular signature, these classifiers must be tested on an independent test or validation set. Here we ask instead, how well the classifiers developed can distinguish between cases with and without relapse in the optimal situation. In all cases, two main clusters are formed, one is enriched in cases with metastases and the other contains only very few of the metastatic cancer samples (Fig. 1). The bar under each clustergram indicates the follow-up information for each case $($ pink $=$ relapse, brown $=$ no relapse). As expected, the classifiers thus work on the dataset on which they have been developed and all eight gene lists yield a similar discrimination. Yet for each classifier, several cases with relapse cluster together with most of the cases without relapse. This could be simply due to a limitation of the single classifier with each misclassifying a different 
Fig. 1 Raw data from 159 consecutive breast cancer cases were obtained from Gene Expression Omnibus (GSE1954). Data were preprocessed by the GCRMA algorithm implemented in Bioconductor. Genes that were significantly associated with the parameter "relapse"

$(P<0.001)$ were selected among the genes listed in the Gene Ontology categories indicated. Hierarchical cluster analysis (Pearson correlation, average linkage) was performed using the genes selected. Cases with relapse are indicated in pink, cases without relapse in brown in the bar beneath each clustergram. The eight annotation categories yield a distinction in two major clusters. Most of the cases with metastases cluster together whereas the other cluster contains only few metastatic cases (the yellow bar indicates the separation between the two major clusters)
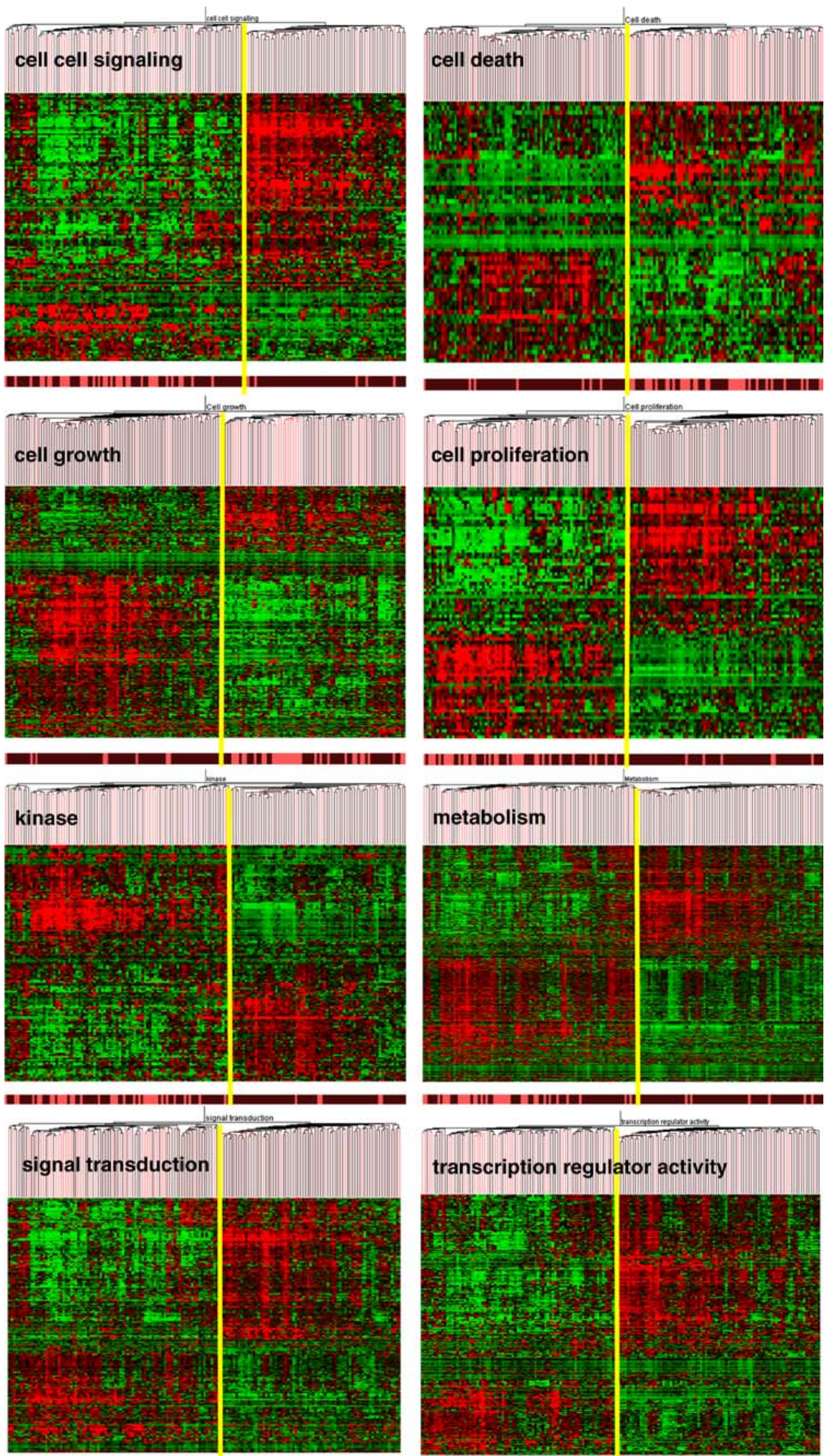

IEIII I II

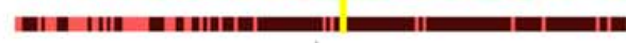

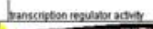

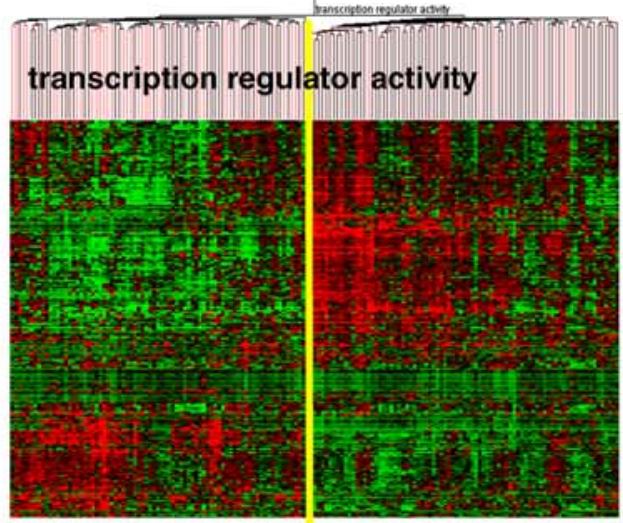

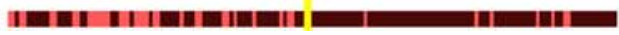


Table 1 Misclassification of breast cancer cases by Gene Ontology based functional clustering

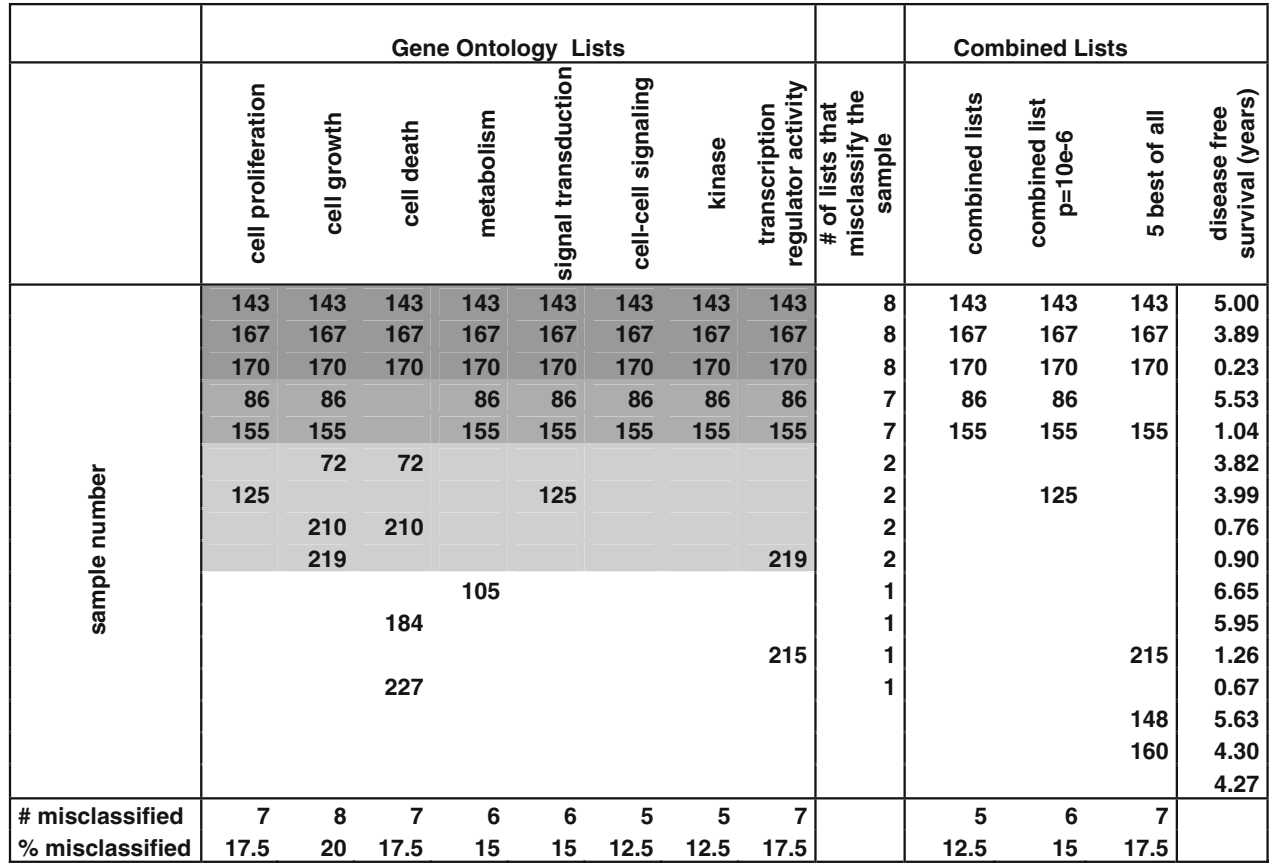

The sample numbers are indicated for cases that were misclassified by the Gene Ontology based gene lists or by combined lists. The gray scale indicates the frequency of misclassifications (dark gray $=8$ of 8 , mid gray $=7$ of 8 , light gray $=2$ of 8 ). The disease free survival is indicated for each case on the right most column. Note that 3 cases are misclassified by clustering with any list and 5 cases are misclassified by 7 of 8 gene lists. Combined list do not perform better. There is no obvious relation between misclassification and time of disease free survival

subset of cases. This would be the case if, for instance, a group of metastatic cancers are similar to the non-metastatic samples as far as proliferation is concerned but not when metabolism is considered. However, when we looked at the samples actually misclassified by the different classifier gene lists we noticed that several cases were misclassified by many or even by all of the eight classifiers (Table 1). Three of forty cases with metastases clustered together with the more benign samples for all of the eight lists and five cases were misclassified by seven out of eight lists. We also tested combinations of the lists using either all of the genes contained in the eight lists (combined lists), only those genes of the eight lists that are associated with relapse with a $P<10^{-6}$ or using the five most strongly associated genes of each list. Again, a similar picture is obtained (Fig. 2): two clusters with a clearly different content of metastatic cases are obtained and the "good prognosis" cluster contains six misclassified cases, among which the five samples that are most frequently misclassified by the single lists. Misclassification could have been expected more easily for cases that showed metastases at very long times after diagnosis, but this is not the case (Table 1).

We then compiled a list of genes that are significantly differently expressed between correctly classified cases with metastases and the five most frequently misclassified cases using a permutation test (Significance Analysis

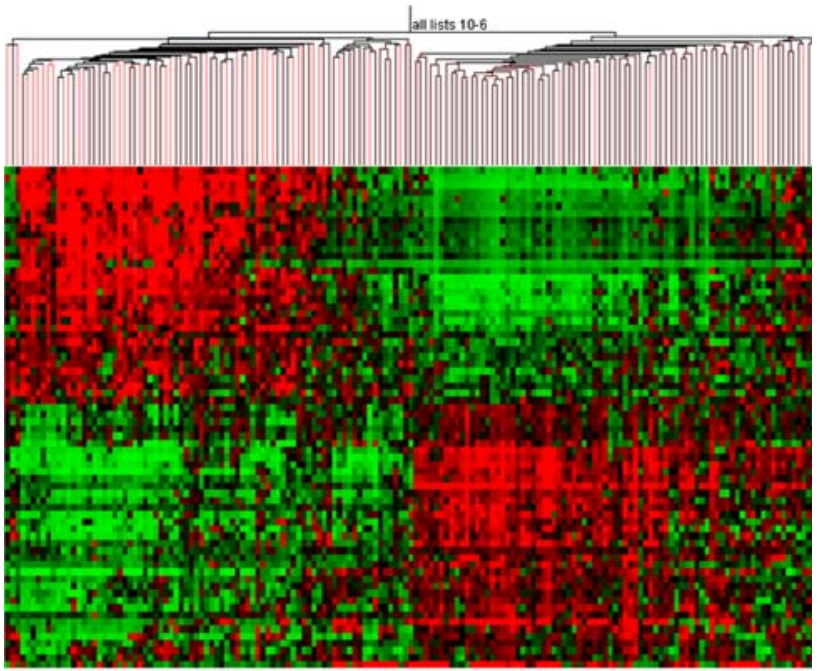

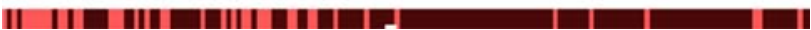

Fig. 2 Hierarchical clustering of 159 breast cancer samples using a gene list containing genes selected from the combination of all eight gene lists used in Fig. 1 containing 1,085 genes. Genes that are associated with the parameter "relapse" with $P$ value below $10^{-6}$ were selected. The combination of all gene lists equally misclassifies a number of cases, among which those misclassified by all the single gene lists

of Microarrays [71]). The genes selected are expressed at similar levels in misclassified cases and in cases without metastases. When we tried to identify genes that are 
expressed at significantly different levels in misclassified cases with metastases and cases without metastasis no gene passed the threshold. The misclassified cases are evidently very similar to tumors that do not metastasize, hence molecular classifiers fail to classify them correctly.

\section{The problem of misclassification}

Intrinsic misclassification could come about through various reasons: (1) annotation errors (erroneously annotated metastases), (2) metastases derived from different clinically non overt primary breast cancers (second primaries), (3) acquisition of the metastatic phenotype by only a very small subpopulation of cells in the primary tumor which would not leave a trace on the expression profile, (4) metastasis of non-metastatic cancers. Although annotation errors can never be totally excluded we believe that they would not occur in as many as $15 \%$ of the cases. Similarly, while non overt second primaries cannot be completely excluded they are expected to account for less than the proportion detected. Acquisition of the metastatic phenotype by only a small subpopulation of the primary tumor cells, or even after the cells have left the primary tumor, is exactly what the multistep carcinogenesis model predicts. The fact that gene expression profiling of the bulk of the primary tumor allows to prognosticate metastasis is a violation of the multistep carcinogenesis theory as Bernards and Weinberg [8] pointed out. These authors proposed that the fate of the tumor is determined right from the initiating event, the transforming mutation, and it might also depend on the cell of origin [46]. These two features, cellular origin and driving mutations, are what microarray profiling is best at detecting. Hence most if not all of the prognostic power of the approach might reside in the correct and exhaustive identification of these two crucial aspects of tumor biology. Yet this does not exclude that, at least in some case, the metastatic phenotype is acquired by an additional mutation by a single cell in the primary tumor or by a cell that, although devoid of metastatic growth characteristics, acquires this potential through mutations. A potential scenario could involve an invasive, anoikis resistant and extravasation competent cell that is able to survive but not to proliferate in a target tissue until a mutation in a receptor gene confers growth factor independent growth. Such a mutation would be rare, since it must occur in the limited number of cells that have formed micrometastases, thus as a consequence the primary tumor would be classified as a "non metastatic" one.

The multistep carcinogenesis model therefore meets a model of probabilistic metastasis that predicts that any invasive cancer can yield metastases but it does so with varying probabilities (being in no case equal to zero). This probability relies on the cell of origin and the driving mutation as well as on the genetic background of the host and the tumor microenvironment, including the host's immune response, inflammation and tumor infiltrate [19]. The acquisition of additional mutations that confer an increased metastatic propensity accounts for the residual metastasis risk that is not derived from the above features and cannot not be predicted. Microarray expression profiling measures, to different extents, all of the above risk modulating factors, with cellular origin and driving mutation probably being the master discriminators that correspond to the main clusters formed by unsupervised hierarchical clustering. Differences in the tumor microenvironment, inflammation, host factors and the like, if they have an influence on the tumor, will leave a trace in the expression profile. The mutation in a subpopulation or, worse, in an already disseminated cell, escapes expression profiling and therefore yields a subfraction of tumors that are "misclassified". This suggests that a relatively acute multistep carcinogenesis model occurs in a set percentage of cases, in the cohort used here approximately $10 \%$, that are likely responsible for metastasis from a primary tumor with a "non-metastatic" phenotype. This would also suggest that the same misclassification would be made by more traditional grading approaches, as the cells with true metastatic potential are quite rare within the primary tumor, thus the histopathological grade would also missclassifly these same tumors.

\section{Improvement of molecular signatures}

This interpretation postulates a theoretical limit to any prognostic procedure that relies on the primary tumor. With a relatively high degree of accurate relapse prognostication, present signatures might already be close to this theoretical limit. But how can we drive them further to actually reach the limit?

In our opinion, three approaches will permit to improve the existing signatures:

- Large sets of samples on which to build and test signatures

- Subtype specific signatures

- Re-introduction of functional information into the signatures

The need for large datasets has been highlighted by the analyses of Ein-Dor and colleagues: in very large datasets the effect of the actual composition of the sample types will be diluted and any possible tumor type will actually be present. Hence, the signatures are expected to become more robust and more generally applicable [67, 68]. 
Subtype specific signatures are expected to have an improved prognostic power since tumors of different subtypes are profoundly different and therefore most likely follow different routes to metastasis. Such signatures become possible if very large datasets are analyzed.

The re-introduction of functional information is expected to yield more robust signatures inasmuch as the genes contained in the signature are mechanistically linked to the process of metastasis. The matrix metalloproteinase 1 (MMP1), for example, has been shown to be functionally linked to metastasis and is contained in the general metastasis signature developed by Ramaswamy et al. [18] and in the metastasis signature obtained from highly metastatic MDA-MB231 mammary breast cancer cells [72]. MMP1 expression shows a very clear expression difference in breast cancer samples [19]. If overexpression of this proteinase could be shown to be necessary for metastasis in a specific subtype of breast cancers, it could become a particularly robust, causally involved marker. The wound healing signature [63] and the invasive gene signature [64] have exploited this functionally oriented approach and were able to show that it is possible to derive valuable prognostic signatures from it. Figure 1 also illustrates that the simple use of functional information already contained in Gene Ontology allows for compiling molecular classifiers. Similarly, Achyrya and colleagues used pathway information to refine the estimation of relapse-free survival and sensitivity to chemotherapy [73]. In addition to an improved prognostication, information on response to chemotherapy can be obtained.

Most probably, a complex functionally defined signature would consist in a meta-signature that has been built by taking into account several functional aspects of metastasis. The use of annotations like Gene Ontology is, however, not sufficient for this approach because the functional information for genes is still relatively limited and mainly depends on the context in which the genes have been analyzed. Experimental evidence of gene function should therefore be collected in a context specific manner before building signatures.

An additional improvement of signatures could be expected from the application of latest generation microarray platforms with more exhaustive (exon rather than transcript coverage) and more robust sets of gene specific probes [23].

\section{MicroRNAs}

Concurrently, new actors in the cellular regulation plot are being investigated. Small non coding RNAs, such as 2123-mer microRNAs that regulate mRNA stability and translatability show important functions in tumor initiation and progression $[74,75]$. Apparently, their expression can be taken as a surrogate of breast cancer subtypes [76] although differences in miRNA expression within the single subtypes cannot be excluded. Integration of miRNA with mRNA expression profiles could eventually further improve molecular signatures. New technologies reveal many more transcripts than previously estimated [77], hence new entries of transcripts or even transcript classes with functional and/or prognostic significance may soon be detected.

\section{Clinical application}

Oncologists are debating over the need or the possibility to integrate information obtained from genomic signatures into the process of treatment decisions. This is already useful in situations where a reasonable doubt on the best treatment persists after classical prognostication, as it could be the case for small, ER-positive, HER2negative, lymphnode negative, low proliferation index (KI67) cases of grade 2 cancers. Since the application of molecular signatures aims at reducing over-treatment, thereby invariably increasing the risk of under-treatment (in the case of misclassification), much will depend on the individual orientation of the patient. Molecular profiling can be offered as an additional source of information if the patient would prefer to avoid chemotherapy, if possible.

Molecular profiling, as a relatively recent technology, still has elevated costs, partly determined by the fact that existing protocols are offered as centralized services. It can be foreseen that with the growing introduction of genomic approaches into the clinical routine, de-centralized devices will become available and lead to cost reductions. Genomics also has the potential to substitute several classical analyses since the correlation between gene expression data and classical analytical methods for prognostic breast cancer markers (estrogen and progesterone receptor and KI67 expression) is excellent (U. Pfeffer, unpublished observations). Hence, it is conceivable that future pathologists will obtain most of their information on a breast cancer sample from a single complex highly standardized genomic assay.

It is important that the screening of breast cancer samples using whole genome platforms continues in order to design better prognostic signatures. This should not be compromised by the application of existing classifiers. Data obtained from validation studies of molecular classifiers (if publicly funded and published on peer reviewed journals) must be made available to the scientific community. 
Acknowledgments We are indebted to Paolo Pronzato, Lucia Del Mastro, Alessandra Gennari and Paolo Bruzzi for helpful discussions. This work was funded by the Ministero della Salute, by the Compagnia di San Paolo and by the Istituto Superiore Sanità (Italia-USA) to UP and AA.

Open Access This article is distributed under the terms of the Creative Commons Attribution Noncommercial License which permits any noncommercial use, distribution, and reproduction in any medium, provided the original author(s) and source are credited.

\section{References}

1. Hanahan D, Weinberg RA (2000) The hallmarks of cancer. Cell 100(1):57-70. doi:10.1016/S0092-8674(00)81683-9

2. Fidler IJ, Kripke ML (1977) Metastasis results from preexisting variant cells within a malignant tumor. Science 197(4306):893895. doi:10.1126/science. 887927

3. Fearon ER, Hamilton SR, Vogelstein B (1987) Clonal analysis of human colorectal tumors. Science 238(4824):193-197. doi:10.1126/science. 2889267

4. Nowell PC (1976) The clonal evolution of tumor cell populations. Science 194(4260):23-28. doi:10.1126/science. 959840

5. Vogelstein B, Fearon ER, Kern SE et al (1989) Allelotype of colorectal carcinomas. Science 244(4901):207-211. doi:10.1126/ science. 2565047

6. Fidler IJ (1990) Critical factors in the biology of human cancer metastasis: twenty-eighth G.H.A. Clowes memorial award lecture. Cancer Res 50(19):6130-6138

7. Fidler IJ, Kripke ML (2003) Genomic analysis of primary tumors does not address the prevalence of metastatic cells in the population. Nat Genet 34(1):23. doi:10.1038/ng0503-23a (author reply 5)

8. Bernards R, Weinberg RA (2002) A progression puzzle. Nature 418(6900):823. doi:10.1038/418823a

9. Gatenby RA, Maini P (2002) Modelling a new angle on understanding cancer. Nature 420(6915):462. doi:10.1038/420462b

10. Edwards, PA (2002) Metastasis: the role of chance in malignancy. Nature 419(6907):559-560. doi:10.1038/419559b (author reply 60 )

11. Player A, Barrett JC, Kawasaki ES (2004) Laser capture microdissection, microarrays and the precise definition of a cancer cell. Expert Rev Mol Diagn 4(6):831-840. doi:10.1586/14737159.4.6.831

12. Langley RR, Fidler IJ (2007) Tumor cell-organ microenvironment interactions in the pathogenesis of cancer metastasis. Endocr Rev 28(3):297-321. doi:10.1210/er.2006-0027

13. Fidler IJ (2002) The organ microenvironment and cancer metastasis. Differentiation 70(9-10):498-505. doi:10.1046/j. 1432-0436.2002.700904.x

14. Fidler IJ (2001) Seed and soil revisited: contribution of the organ microenvironment to cancer metastasis. Surg Oncol Clin N Am 10(2):257-269 (vii-viiii)

15. Albini A, Sporn MB (2007) The tumour microenvironment as a target for chemoprevention. Nat Rev Cancer 7(2):139-147. doi:10.1038/nrc2067

16. Liotta LA, Kohn EC (2001) The microenvironment of the tumour-host interface. Nature 411(6835):375-379. doi:10.1038/ 35077241

17. Sleeman JP, Cremers N (2007) New concepts in breast cancer metastasis: tumor initiating cells and the microenvironment. Clin Exp Metastasis 24(8):707-715. doi:10.1007/s10585-007-9122-6

18. Ramaswamy S, Ross KN, Lander ES et al (2003) A molecular signature of metastasis in primary solid tumors. Nat Genet 33(1):49-54. doi:10.1038/ng1060
19. Albini A, Mirisola V, Pfeffer U (2008) Metastasis signatures: genes regulating tumor-microenvironment interactions predict metastatic behavior. Cancer Metastasis Rev 27(1):75-83. doi:10.1007/s10555-007-9111-x

20. Patocs A, Zhang L, Xu Y et al (2007) Breast-cancer stromal cells with TP53 mutations and nodal metastases. N Engl J Med 357(25):2543-2551. doi:10.1056/NEJMoa071825

21. Chen JJ, Delongchamp RR, Tsai CA et al (2004) Analysis of variance components in gene expression data. Bioinformatics (Oxford, England) 20(9):1436-1446. doi:10.1093/bioinformatics/ bth118

22. Mecham BH, Klus GT, Strovel J et al (2004) Sequence-matched probes produce increased cross-platform consistency and more reproducible biological results in microarray-based gene expression measurements. Nucleic Acids Res 32(9):e74. doi:10.1093/ nar/gnh071

23. Kapur K, Xing Y, Ouyang Z et al (2007) Exon arrays provide accurate assessments of gene expression. Genome Biol 8(5):R82. doi:10.1186/gb-2007-8-5-r82

24. Symmans WF, Ayers M, Clark EA et al (2003) Total RNA yield and microarray gene expression profiles from fine-needle aspiration biopsy and core-needle biopsy samples of breast carcinoma. Cancer 97(12):2960-2971. doi:10.1002/cncr.11435

25. Sotiriou C, Powles TJ, Dowsett M et al (2002) Gene expression profiles derived from fine needle aspiration correlate with response to systemic chemotherapy in breast cancer. Breast Cancer Res 4(3):R3. doi:10.1186/bcr433

26. Pusztai L, Ayers M, Stec J et al (2003) Gene expression profiles obtained from fine-needle aspirations of breast cancer reliably identify routine prognostic markers and reveal large-scale molecular differences between estrogen-negative and estrogenpositive tumors. Clin Cancer Res 9(7):2406-2415

27. Mutter GL, Zahrieh D, Liu C et al (2004) Comparison of frozen and RNALater solid tissue storage methods for use in RNA expression microarrays. BMC Genomics 5(1):88. doi:10.1186/ 1471-2164-5-88

28. Goldmann T, Flohr AM, Murua Escobar H et al (2004) The HOPE-technique permits Northern blot and microarray analyses in paraffin-embedded tissues. Pathol Res Pract 200(7-8):511515. doi:10.1016/j.prp.2004.04.008

29. Perou CM, Sorlie T, Eisen MB et al (2000) Molecular portraits of human breast tumours. Nature 406(6797):747-752. doi:10.1038/ 35021093

30. Weigelt B, Hu Z, He X et al (2005) Molecular portraits and 70gene prognosis signature are preserved throughout the metastatic process of breast cancer. Cancer Res 65(20):9155-9158. doi:10.1158/0008-5472.CAN-05-2553

31. Weigelt B, Glas AM, Wessels LF et al (2003) Gene expression profiles of primary breast tumors maintained in distant metastases. Proc Natl Acad Sci USA 100(26):15901-15905. doi:10.1073/ pnas. 2634067100

32. Feng Y, Sun B, Li X et al (2007) Differentially expressed genes between primary cancer and paired lymph node metastases predict clinical outcome of node-positive breast cancer patients. Breast Cancer Res Treat 103(3):319-329. doi:10.1007/s10549006-9385-7

33. Vecchi M, Nuciforo P, Romagnoli S et al (2007) Gene expression analysis of early and advanced gastric cancers. Oncogene 26(29):4284-4294. doi:10.1038/sj.onc. 1210208

34. Sorlie T, Perou CM, Tibshirani R et al (2001) Gene expression patterns of breast carcinomas distinguish tumor subclasses with clinical implications. Proc Natl Acad Sci USA 98(19):1086910874. doi:10.1073/pnas. 191367098

35. Gruvberger S, Ringner M, Chen Y et al (2001) Estrogen receptor status in breast cancer is associated with remarkably distinct gene expression patterns. Cancer Res 61(16):5979-5984 
36. Sotiriou C, Neo SY, McShane LM et al (2003) Breast cancer classification and prognosis based on gene expression profiles from a population-based study. Proc Natl Acad Sci USA 100(18):10393-10398. doi:10.1073/pnas. 1732912100

37. Hu Z, Fan C, Oh DS et al (2006) The molecular portraits of breast tumors are conserved across microarray platforms. BMC Genomics 7:96. doi:10.1186/1471-2164-7-96

38. Kapp AV, Tibshirani R (2007) Are clusters found in one dataset present in another dataset? Biostatistics (Oxford, England) 8(1):9-31. doi:10.1093/biostatistics/kxj029

39. Kapp AV, Jeffrey SS, Langerod A et al (2006) Discovery and validation of breast cancer subtypes. BMC Genomics 7:231. doi:10.1186/1471-2164-7-231

40. Vanden Bempt I, Drijkoningen M, De Wolf-Peeters C (2007) The complexity of genotypic alterations underlying HER2-positive breast cancer: an explanation for its clinical heterogeneity. Curr Opin Oncol 19(6):552-557

41. Menard S, Pupa SM, Campiglio M et al (2003) Biologic and therapeutic role of HER2 in cancer. Oncogene 22(42):6570 6578. doi:10.1038/sj.onc. 1206779

42. Richardson AL, Wang ZC, De Nicolo A et al (2006) X chromosomal abnormalities in basal-like human breast cancer. Cancer Cell 9(2):121-132. doi:10.1016/j.ccr.2006.01.013

43. Hedenfalk I, Duggan D, Chen Y et al (2001) Gene-expression profiles in hereditary breast cancer. N Engl J Med 344(8):539548. doi:10.1056/NEJM200102223440801

44. Fan C, Oh DS, Wessels L et al (2006) Concordance among geneexpression-based predictors for breast cancer. N Engl J Med 355(6):560-569. doi:10.1056/NEJMoa052933

45. Perreard L, Fan C, Quackenbush JF et al (2006) Classification and risk stratification of invasive breast carcinomas using a realtime quantitative RT-PCR assay. Breast Cancer Res 8(2):R23. doi:10.1186/bcr1399

46. Gupta PB, Kuperwasser C, Brunet JP et al (2005) The melanocyte differentiation program predisposes to metastasis after neoplastic transformation. Nat Genet 37(10):1047-1054. doi:10.1038/ ng 1634

47. Dupuy A, Simon RM (2007) Critical review of published microarray studies for cancer outcome and guidelines on statistical analysis and reporting. J Natl Cancer Inst 99(2):147-157. doi:10.1093/jnci/djk018

48. Roukos DH, Briasoulis E (2007) Individualized preventive and therapeutic management of hereditary breast ovarian cancer syndrome. Nat Clin Pract Oncol 4(10):578-590. doi:10.1038/ ncponc0930

49. Miller LD, Liu ET (2007) Expression genomics in breast cancer research: microarrays at the crossroads of biology and medicine. Breast Cancer Res 9(2):206. doi:10.1186/bcr1662

50. Lonning PE, Sorlie T, Borresen-Dale AL (2005) Genomics in breast cancer-therapeutic implications. Nat Clin Pract Oncol 2(1):26-33. doi: $10.1038 /$ ncponc0072

51. Lonning PE, Knappskog S, Staalesen V et al (2007) Breast cancer prognostication and prediction in the postgenomic era. Ann Oncol 18(8):1293-1306. doi:10.1093/annonc/mdm013

52. Driouch K, Landemaine T, Sin S et al (2007) Gene arrays for diagnosis, prognosis and treatment of breast cancer metastasis. Clin Exp Metastasis 24(8):575-585. doi:10.1007/s10585-007-9110-x

53. Brenton JD, Carey LA, Ahmed AA et al (2005) Molecular classification and molecular forecasting of breast cancer: ready for clinical application? J Clin Oncol 23(29):7350-7360. doi:10.1200/JCO.2005.03.3845

54. Rennstam K, Hedenfalk I (2006) High-throughput genomic technology in research and clinical management of breast cancer. Molecular signatures of progression from benign epithelium to metastatic breast cancer. Breast Cancer Res 8(4):213. doi:10.1186/ bcr 1528
55. Cheang MC, van de Rijn M, Nielsen TO (2008) Gene expression profiling of breast cancer. Annu Rev Pathol 3:67-97. doi:10.1146/annurev.pathmechdis.3.121806.151505

56. Paik S, Shak S, Tang G et al (2004) A multigene assay to predict recurrence of tamoxifen-treated, node-negative breast cancer. $\mathrm{N}$ Engl J Med 351(27):2817-2826. doi:10.1056/NEJMoa041588

57. Sparano JA, Paik S (2008) Development of the 21-gene assay and its application in clinical practice and clinical trials. J Clin Oncol 26(5):721-728. doi:10.1200/JCO.2007.15.1068

58. van 't Veer LJ, Dai H, van de Vijver MJ et al (2002) Gene expression profiling predicts clinical outcome of breast cancer. Nature 415(6871):530-536. doi:10.1038/415530a

59. van de Vijver MJ, He YD, van't Veer LJ et al (2002) A geneexpression signature as a predictor of survival in breast cancer. $\mathrm{N}$ Engl J Med 347(25):1999-2009. doi:10.1056/NEJMoa021967

60. Eden P, Ritz C, Rose C et al (2004) "Good Old" clinical markers have similar power in breast cancer prognosis as microarray gene expression profilers. Eur J Cancer 40(12):1837-1841. doi: 10.1016/j.ejca.2004.02.025

61. Cardoso F, Van't Veer L, Rutgers E et al (2008) Clinical application of the 70-gene profile: the MINDACT trial. J Clin Oncol 26(5):729-735. doi:10.1200/JCO.2007.14.3222

62. Foekens JA, Atkins D, Zhang Y et al (2006) Multicenter validation of a gene expression-based prognostic signature in lymph node-negative primary breast cancer. J Clin Oncol 24(11):16651671. doi:10.1200/JCO.2005.03.9115

63. Chang HY, Nuyten DS, Sneddon JB et al (2005) Robustness, scalability, and integration of a wound-response gene expression signature in predicting breast cancer survival. Proc Natl Acad Sci USA 102(10):3738-3743. doi:10.1073/pnas.0409462102

64. Liu R, Wang X, Chen GY et al (2007) The prognostic role of a gene signature from tumorigenic breast-cancer cells. N Engl J Med 356(3):217-226. doi:10.1056/NEJMoa063994

65. Loi S, Haibe-Kains B, Desmedt C et al (2007) Definition of clinically distinct molecular subtypes in estrogen receptor-positive breast carcinomas through genomic grade. J Clin Oncol 25(10):1239-1246. doi:10.1200/JCO.2006.07.1522

66. Ross JS, Hatzis C, Symmans WF et al (2008) Commercialized multigene predictors of clinical outcome for breast cancer. Oncol 13(5):477-493. doi:10.1634/theoncologist.2007-0248

67. Ein-Dor L, Kela I, Getz G et al (2005) Outcome signature genes in breast cancer: is there a unique set? Bioinformatics (Oxford, England) 21(2):171-178. doi:10.1093/bioinformatics/bth469

68. Ein-Dor L, Zuk O, Domany E (2006) Thousands of samples are needed to generate a robust gene list for predicting outcome in cancer. Proc Natl Acad Sci USA 103(15):5923-5928. doi: 10.1073/pnas.0601231103

69. Pawitan Y, Bjohle J, Amler L et al (2005) Gene expression profiling spares early breast cancer patients from adjuvant therapy: derived and validated in two population-based cohorts. Breast Cancer Res 7(6):R953-R964. doi:10.1186/bcr1325

70. Day-Richter J, Harris MA, Haendel M et al (2007) OBO-Edit an ontology editor for biologists. Bioinformatics (Oxford, England) 23(16):2198-2200. doi:10.1093/bioinformatics/btm112

71. Tusher VG, Tibshirani R, Chu G (2001) Significance analysis of microarrays applied to the ionizing radiation response. Proc Natl Acad Sci USA 98(9):5116-5121. doi:10.1073/pnas.091062498

72. Minn AJ, Gupta GP, Siegel PM et al (2005) Genes that mediate breast cancer metastasis to lung. Nature 436(7050):518-524. doi:10.1038/nature03799

73. Acharya CR, Hsu DS, Anders CK et al (2008) Gene expression signatures, clinicopathological features, and individualized therapy in breast cancer. J Am Med Assoc 299(13):1574-1587. doi:10.1001/jama.299.13.1574

74. Calin GA, Croce CM (2006) MicroRNA signatures in human cancers. Nat Rev Cancer 6(11):857-866. doi:10.1038/nrc1997 
75. Lowery AJ, Miller N, McNeill RE et al (2008) MicroRNAs as prognostic indicators and therapeutic targets: potential effect on breast cancer management. Clin Cancer Res 14(2):360-365. doi:10.1158/1078-0432.CCR-07-0992

76. Blenkiron C, Goldstein LD, Thorne NP et al (2007) MicroRNA expression profiling of human breast cancer identifies new markers of tumor subtype. Genome Biol 8(10):R214. doi: 10.1186/gb-2007-8-10-r214

77. Kapranov P, Cheng J, Dike S et al (2007) RNA maps reveal new RNA classes and a possible function for pervasive transcription. Science 316(5830):1484-1488. doi:10.1126/science.1138341 